\title{
Precision of scoring radiation-induced chromosomal aberrations and micronuclei by unexperienced scorers
}

\author{
Maciej Gałecki ${ }^{1}$, Adrianna Tartas ${ }^{1}$, Agata Szymanek ${ }^{2}$, Emma Sims $^{3}$, LovisaLundholm ${ }^{4}$, Alice Sollazzo ${ }^{4}$, \\ Lei Cheng ${ }^{4}$, Yohei Fujishima ${ }^{5}$, Mitsuaki A. Yoshida ${ }^{5}$, Jarosław Żygierewicz ${ }^{1}$, Andrzej Wojcik ${ }^{4,6}$, Beata Brzoz \\ owska-Wardecka*1 \\ ${ }^{1}$ Biomedical Physics Division, Faculty of Physics, University of Warsaw, Warsaw, ${ }^{\text {Poland }}$ \\ ${ }^{2}$ Ardigen, Krakow, Poland

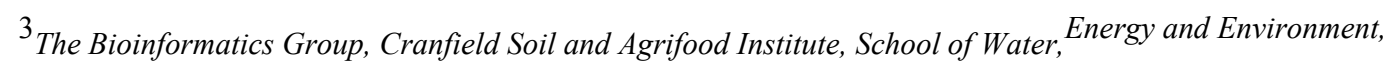 \\ Cranfield University, Bedford MK43 OAL, UK \\ ${ }^{4}$ Department of Molecular Biosciences, The Wenner-Gren Institute, Centre for Radiation Protection Res \\ earch, Stockholm University, Sweden \\ ${ }^{5}$ Department of Radiation Biology, Institute of Radiation Emergency Medicine, Hirosaki University, Japan \\ $6_{\text {Institute of Biology, Jan Kochanowski University, Kielce, Poland }}$
}

*Corresponding author: Beata Brzozowska-Wardecka, beata.brzozowska@fuw.edu.pl

Biomedical Physics, Division, Faculty of Physics, University of Warsaw, 5 PasteuraStreet, 02-093 Warsaw, Pol

and

Maciej Galecki is a PhD student at the Biomedical Physics Division, Faculty of Physics, University of Warsaw. His research area of interest is computer vision and machine learning applications in medicine and psychology. He is also committed to developing a tool that enables automatic analysis of images of cell colonies obtained in physical and biological experiments.

Adrianna Tartas is a PhD student at the Biomedical Physics Division, Faculty of Physics, University of Warsaw. Her main research area is Monte Carlo modeling to describe the 
processes of formation of repair foci as a result of various types of ionizing radiation on human cells.

Agata Szymanek works as a bioinformatician in a Research and Development group of Ardigen, specializing in genomics and data analysis. She holds a double degree in Biotechnology and Applied Bioinformatics. During her engineering thesis, she was applying statistical methods for GWAS study in radiosensitivity, which led her to participate in the CELOD (cellular effects of ionizing radiation) course. She was also collaborating with the London School of Hygiene and Tropical Medicine, investigating the genetic background of parasitic drug resistance. Whilst working in the Cancer Center Amsterdam, she was conducting a statistical analysis on retinoblastoma patients' transcriptomes, and she participated in numerous training opportunities, including both the European Bioinformatics Institute and the Netherlands Bioinformatics and Systems Biology research school courses. Currently, she is working in the field of genomics and human microbiome research.

Emma Sims is a PhD student at Cranfield University and part of the Bioinformatics Group in Agrifood. She holds degrees in Computer Science, Mathematics, and Applied Bioinformatics; specialising in statistics, machine learning, and software development. Previously she has developed an R package which can simultaneously analyse and compare various modelling techniques for a range of proteomics input data. Her current projects involve developing pattern recognition algorithms within images, and developing cloud computing web platforms to analyse the metabolomics data of fresh food items.

Lovisa Lundholm, $\mathrm{PhD}$, is a researcher in the field of radiation biology at the Stockholm University. She has a background within molecular biology and cancer research. Her work focuses on the impact of chromatin condensation on the response of cells to radiations of different qualities.

Alice Sollazzo defended her PhD in Molecular Bioscience, Radiobiology, in 2017 at The Department of Molecular Biosciences, The Wenner-Gren Institute (MBW), Stockholm University, Stockholm Sweden. Her work focuses on gaining new insight on the molecular mechanisms behind the repair of DNA damage caused by exposure to mixed- fields of low and high LET radiation.

Lei Cheng is a PhD student in the field of radiation biology in the Department of Molecular Biosciences, The Wenner-Gren Institute (MBW) at the Stockholm University. Cheng focuses on studying the cellular effects of combined exposure to radiations of different qualities. 
Yohei Fujishima, M.Sc., is a PhD student in Department of Radiation Biology, Institute of Radiation Emergency Medicine (IREM/HU), Hirosaki University. His research field is cytogenetic biodosimetry including optimizations and developing methodologies of blood culture for biodosimetry.

Mitsuaki A. Yoshida, $\mathrm{PhD}$, is a professor at the Department of Radiation Biology, Institute of Radiation Emergency Medicine (IREM/HU), Hirosaki University. His professional fields are molecular cytogenetics including chromosome analysis by banding methods and chromosome dose assessment in the radiation exposure accident. Also, he is contributing to the establishment of the system for preparedness and response of radiation emergency medicine. After the accident at the Fukushima Nuclear Power Station due to the 2011 East Japan great earthquake, he continues to support Fukushima using the chromosome analysis method in order to investigate the effect of radiological substances released from Fukushima Dai-Ichi NPP. He is also a member of WHO BioDoseNet and the Chromosome Network Council in Japan.

Jaroslaw Żygierewicz, $\mathrm{PhD}$, is an Assistant Professor at the Biomedical Physics Division, Faculty of Physics, University of Warsaw. His research interests concern time-frequency analysis of EEG and MEG signals. He developed methodology for statistical analysis of event related synchronization and desynchronization in EEG and MEG. He is involved in designing brain computer interfaces, validation of psychological models of emotions and working memory. His research encompasses also exploratory data analysis.

Andrzej Wojcik, $\mathrm{PhD}$, is professor of radiation biology at the Stockholm University. He focuses on studying the cellular effects of radiation, with special focus on combined exposure to radiations of different qualities. He is also interested in developing and validating cytogenetic tools for biological dosimetry.

Beata Brzozowska-Wardecka, PhD, works in the Biomedical Physics Division at Faculty of Physics, University of Warsaw and her scientific interests concern medical physics, radiobiology in particular. She joined the mixed beam effect for cancer risk project at Stockholm University. She now works with PARTRAC codes (in cooperation with W. Friendland, Helmholtz Centre) to model DNA damage and repair in cells exposed to ionizing radiation. 


\title{
Precision of scoring radiation-induced chromosomal aberrations and micronuclei by unexperienced scorers
}

\author{
Purpose: Dose assessment plays an important role in case of radiological \\ accidents and can be performed by scoring structural changes of chromosome \\ morphology induced in cells by ionizing radiation. The results of such a test are \\ biased by scorer experience, therefore simple to learn assays are recommended to \\ be used when fast analysis of large amount of data is needed. The aim of this \\ study was to compare the performance of two radiobiological assays - \\ chromosomal aberrations and micronuclei - by unexperienced scorers with the \\ reference values generated by an expert.
}

Materials and methods: Each participant of an EU-funded two-week radiobiology course was asked to score Chinese hamster ovary cells exposed to gamma radiation up to $4 \mathrm{~Gy}$. The congruence of students' and expert's scores at each dose and the coherence of the dose-response curve parameters between the students were investigated.

Results: Micronucleus test tended to be faster and easier to learn than scoring chromosomal aberrations. However, both assays carried out by inexperienced students showed reasonable dose-response curves.

Conclusions: In the case of a large radiological accident involving many casualties, the unexperienced scorers would support the process of biodosimetric triage by cytogenetic biological dosimetry.

Keywords: chromosomal aberrations; micronuclei; ionizing radiation; radiobiological assays

\section{Introduction}

Analysis of cytogenetic damage remains a basic endpoint for studying the effects of ionizing radiation in cells. Cytogenetic damage can be visualized as structural changes of chromosome morphology called chromosomal aberrations (CA) or as micronuclei (MN) which are small-sized nuclei containing lagging chromosomes or chromosomal fragments. Radiation-induced CA were initially studied in plant cells (Lea and Catcheside 1942) and their identification and analysis in mammalian cells only became 
possible following the development of appropriate cell culturing and harvesting methods (Holmquist 1987; Tjio and Levan 1956). In 1962 Bender and Gooch discovered that the quantitative analysis of CA in peripheral blood lymphocytes can serve as a reliable retrospective dosimeter (Bender and Gooch 1962). Subsequent work established the CA test as the gold standard of biological dosimetry (Ainsbury et al. 2011; IAEA 2011; Romm et al. 2009). MN were observed by Howell as early as 1891 and by Jolly in 1907 (Müller and Streffer 1994), but their application in mammalian cell radiation biology was pioneered by Countryman and Heddle in 1976 (Countryman and Heddle 1976). The effective use of the MN assay for quantifying radiation-induced DNA damage was made possible by Fenech and Morley who discovered that cytochalasin B prevents cytokinesis while allowing karyokinesis (Fenech and Morley 1986). The cytokinesis block micronucleus (CBMN) assay is routinely used for biological dosimetry in parallel or instead of the chromosomal aberration assay (IAEA 2011).

It is intuitive to assume that $\mathrm{MN}$ are easier to score and the assay faster to learn than the CA assay. The reason for this is that various types of CA exist (Savage 1975) which are sometimes difficult to discern, especially when the chromosomal morphology is poor. In contrast, $\mathrm{MN}$ are easy to recognize and clear criteria exist for their identification (Fenech 2000, 2007). But is scoring of CA really more difficult to learn than that of MN? The question is far from academic in view of the current threat of large-scale radiological emergencies, where triage biological dosimetry will quickly have to be performed on a large number of exposed or potentially exposed people (Lloyd et al. 2000; Wojcik et al. 2010). Although automation of biodosimetric tools and networking of laboratories have been suggested as a solution to cope with a large number of cases to be analyzed (Blakely et al. 2009; Kulka et al. 2017; Wojcik et al. 
2017), situations may arise when neither automation nor networking is available. Then it may also be necessary to quickly educate scorers and the question arises as to which assay should be prioritized (Ainsbury et al. 2011, Wojcik et al. 2017).

A possibility to compare the learning outcome of both assays was offered by a two-week radiobiology course CELOD (cellular effects of ionizing radiation introduction to radiation biology), funded by the European Joint Program CONCERT (http://www.concert-h2020.eu/en) which was held at the Stockholm University in spring of 2016. A total of 33 participants of whom 32 never before scored CA or MN were given two introductory lectures about the assays and then given coded microscopic slides with Chinese hamster ovary cells (CHO-K1) fixed for $\mathrm{CA}$ and $\mathrm{MN}$ analysis. The cells received incrementing doses of gamma radiation, each course participant was given access to a light microscope and was asked to construct a CA and a $\mathrm{MN}$ dose response curve. The slide codes were broken after completion of scoring and the scoring outcome was analyzed.

The aim of this paper was to report the outcome of the course with focus on how efficiently non-experienced people can score CA and $\mathrm{MN}$ after a basic introduction, and how well their estimate of dose response curves adheres to those of an experienced scorer. Data was processed statistically and to avoid the misuse of statistical tests (Greenland et al. 2016) each step of the statistical procedure is described in detail.

\section{Materials and methods}

\section{Course participants}

The course was attended by 33 participants ( 11 females and 22 males), of which 16 were regular Stockholm University biology students at the master level and 17 were international participants, mainly $\mathrm{PhD}$ students of biological sciences. The median age 
was 26. Except for one PhD student, none of the participants had any experience with cytogenetic analyses. The PhD student Yohei Fujishima has been working in the field of radiation cytogenetics since 6 years and published a number of relevant papers (Fujishima et al 2019; Ariyoshi et al. 2018a; Ariyoshi et al. 2018b; Takino et al 2017; Ariyoshi et al. 2016). He is referred to as the 'expert', while the unexperienced scorers are referred to as 'students'.

\section{Cells, irradiation, harvest and scoring of microscopic slides}

Chinese hamster ovary (CHO-K1) cells were grown in McCoy's 5A medium supplemented with 10\% fetal bovine serum, 1\% penicillin-streptomycin (all from Sigma-Aldrich, Stockholm, Sweden) at $37{ }^{\circ} \mathrm{C}$ in a humidified atmosphere with $5 \% \mathrm{CO}_{2}$. $\mathrm{CHO}$ were maintained as exponentially growing monolayer cultures in $25 \mathrm{~cm}^{2}$ plastic flasks.

For CA analysis cells were exposed to gamma radiation $\left({ }^{137} \mathrm{Cs}\right.$ source, dose rate $0.39 \mathrm{~Gy} / \mathrm{min}$, Scanditronix, Sweden) at doses of 0, 0.5, 1.0, 2.0, 3.0 and $4.0 \mathrm{~Gy} .8$ hours later colcemid at a final concentration of $0.1 \mu \mathrm{g} / \mathrm{ml}$ was added to each culture and kept for 2 h. Cells were harvested as described in (Johannes and Obe 1991). At least 6 microscopic slides per dose were prepared, air dried, stained with Giemsa and coded.

For $\mathrm{MN}$ analysis cells were exposed to gamma radiation $\left({ }^{137} \mathrm{Cs}\right.$ source, dose rate $0.39 \mathrm{~Gy} / \mathrm{min}$, Scanditronix, Sweden) at doses of 0, 0.5, 1.0, 2.0, and 3.0 Gy. Immediately thereafter cytochalasin B was added at a final concentration of $5.6 \mu \mathrm{g} / \mathrm{ml}$ and cells were harvested $22 \mathrm{~h}$ later as described in Cheng et al. (2015). At least 6 microscopic slides per dose were prepared, air dried, stained with Giemsa and coded.

Each participant was asked to score 50 mitotic cells per dose point for the CA dose response curve and 500 binucleated cells (BNC) per dose point for the $\mathrm{MN}$ dose response curve. Scoring outcome was registered on dedicated scoring sheets. For CA, 
each aberration was categorized according to Savage (1975) as: chromosome or chromatid break (terminal deletion), dicentric, centric ring, double or single minute (interstitial deletion), chromatid-type interchromosomal exchange, sister union or gap (achromatic lesion) (Fig. 1). For calculating the ratios of chromosome to chromatid-type aberrations, dicentrics and rings were classified as chromosome-type CA and interchromosomal exchanges and chromatid breaks were classified as chromatid-type CA. BNC were classified according to the number of contained MN. The order of scored slides was random. The slide codes were broken after completing the analysis. Not all participants scored all slides: 30 participants scored a complete set of CA slides and 27 participants scored a complete set of MN slides.

During the first 3 days of analysis the participants received active support from the course assistants both regarding the technique of light microscopy and the identification of CA and MN. One participant was experienced in cytogenetic scoring and he also offered assistance. He scored all CA and MN slides and his results were treated as a reference.

\section{Statistical analysis}

The distributions of CA and of MN data were tested by means of Kolmogorov-Smirnov (KS) test against the reference Gaussian and Poisson distribution, and additionally, Shapiro-Wilk test was performed to verify the normality of distribution, as it has more power than KS.

Two criteria were applied to analyze the effectiveness of training. First, the congruence of students' and experts scores at each dose was assessed. A 95\% confidence interval (CI) for the students' score was estimated for each dose value by means of the bootstrap method (Bickel and Freedman 1981). The scores were considered as congruent if the CI included the expert's score. 
The second criterion was the coherence of the dose-response curve parameters between the students. The dose-response relation was assumed to be linear:

$$
y=a D+b
$$

where $y$ - score, $a$ - slope and $b$ - intercept. Parameters $a$ and $b$ were estimated by linear regression for the set of scores of each student separately. To estimate the goodness of the fit the $R^{2}$ and $C h i^{2}$ tests were used. The set of slopes were summarized by the mean value and standard deviation.

The coefficient of variation $(\mathrm{CV})$ is defined as $100(\sigma / \mu)$, where $\sigma$ is the standard deviation and $\mu$ - the mean. It is a measure of the relative error. It was calculated for the estimated parameters allowing comparison between CA and $\mathrm{MN}$ assays. High values of the $\mathrm{CV}$ for the slope indicate that there is little consistency among scorers in estimating the dose-response relation. In other words, the higher the CV value the less reliable the results. Additionally, the $\mathrm{CV}$ was calculated for each type of chromosomal aberration. High values of $\mathrm{CV}$ indicate that the aberration is difficult to identify.

\section{Results}

Student scores of chromosomal aberrations and micronuclei were compared with the reference values to determine precision of both radiobiological assays in the hands of unexperienced observers.

We obtained a different type of statistical distribution for each dose value, as shown in Table A1 in the Appendix A. The CA scores for control, i.e. the nonirradiated cells were Poisson-distributed. In contrast, all chromosomal aberrations detected in irradiated cells, tended to be overdispersed and the Shapiro-Wilk test rejected the hypothesis of normal distribution $(\mathrm{p}<0.05)$.

In the case of $\mathrm{MN}$ scoring, all data followed a normal distribution according to the Kolmogorov-Smirnov test, but the Shapiro-Wilk test rejected that hypothesis. 


\section{Chromosomal aberrations}

Each participant was asked to score all observed chromosomal aberrations and mark the type of each observation according to Fig. 1.

The $95 \%$ confidence intervals for sets of students' scores of total numbers of CA are shown in Fig. 2 together with the scores of the expert.

The expert's scores were within the CI 95\% for doses up to 2 Gy. The students' scores were not congruent with the expert's scores for the two highest doses. This could be caused by the fact that the complexity and level of chromosomal rearrangements increase with the dose, thus making scoring difficult. The quality of chromosomal spreads is also often worse after high doses as compared to low doses. In order to examine more closely the differences observed for high doses, the same calculation procedures were repeated for each type of chromosomal aberration separately. The rational for doing so was to identify aberrations where the students showed the highest degree of disagreement with the expert. The results are shown in Fig.3.

The confidence interval width for student observations increased with increasing dose for most CA types. The highest discrepancy between the students' results was detected for chromatid breaks and interchromosomal exchanges. The largest difference between the students and the expert was observed for chromosome and chromatid gaps. Moreover, students' scores of gaps and chromatid breaks did not increase with the dose. Furthermore, the expert systematically scored fewer rings and sister unions than the students.

To study total CA induction as a function of dose, the individual results were fitted to a linear function. The linear regression parameters for the experts' measurements were: $a=(0.418 \pm 0.050) / \mathrm{Gy}$ and $b=-0.02 \pm 0.11\left(C h i^{2}=0.33, R^{2}\right.$ $=0.95)$. 
Regression parameters: $a$ and $b$ were also estimated for each student (see Tab. B1 in the Appendix B). The experts' dose-response line superposed on the individual students' dose-response lines is presented in Fig. 4.

The distribution of the slope values of all students is illustrated by a histogram in Fig. 5. The mean value of $a$ equals to 0.186 and the standard deviation to 0.081 .

Additionally, in an attempt to check which type of chromosomal aberration was associated with the largest level of disagreement among the scorers, coefficients of variation for each aberration type were calculated from the results of all scorers and shown in Tab.1. The lowest CV was obtained for chromatid breaks and the highest for gaps and sister unions. The other aberration types had similar CV values.

\section{Micronuclei}

Analogously to CA analysis, all calculation procedures were performed for the $\mathrm{MN}$ assay, i.e. CI calculations (Fig. 6), dose-response regression and CV estimation (Table 2). The regression parameters for the dose-response curve for MN assay performed by the expert were: $a=(223 \pm 14) / \mathrm{Gy}$ and $b=7 \pm 24$. The regression was also performed for all the students individually. The obtained slope values with the corresponding $C h i^{2}$ and $R^{2}$ values are shown in Tab. B2 in the Appendix B. The mean value of the students' slope parameter and its standard deviation was $172 \pm 62$. The regression lines are shown together with all MN observations in Fig. 7. The distribution of slopes is shown in Fig. 8.

\section{Comparison of chromosomal aberrations and micronuclei}

As mentioned before, there were two criteria used for comparing the $\mathrm{MN}$ and $\mathrm{CA}$ assays. The first was the assessment of congruence of $\mathrm{CA}$ and $\mathrm{MN}$ frequencies for a given dose achieved by the students and by the expert. It was checked if the expert's 
scores are within the 95\% CI of student data as presented in Fig. 2 and Fig. 6. While the agreement between these two types of MN scores was observed in the whole range of doses, CA observations were not consistent in cell samples exposed to doses in excess of $2 \mathrm{~Gy}$.

The second criterion was to investigate the dose-response relationships as means to evaluate possible systematic bias of the observers. A bias would be indicated by similar slopes and different intercepts. As shown in the appendix $\mathrm{B}$, the CA intercept $b$ was equal to 0 within calculated uncertainties for all students and the expert.

Coefficients of variation based on the mean slope value and its standard deviation were calculated for both radiobiological assays according to the definition given in Materials and methods. Those values with estimated uncertainties are given in Tab. 2.

The results show a statistically significant difference with $\mathrm{p}<0.025$ (according to F-test of variance equality) between $\mathrm{CV}$ from both assays. The variation of doseresponse curve slopes for $\mathrm{MN}$ scoring is smaller than the variation for CA test which is visible clearly as a wider Gaussian distribution for the CA test.

\section{Discussion}

In the case of a large radiological accident involving many casualties, the advantage of having multiple scorers would greatly speed up the process of biodosimetric triage by cytogenetic biological dosimetry. Given the fact that experienced scorers are rare (Wojcik et al. 2010), the acquisition of multiple scorers will require hiring people who are novices to cytogenetic scoring. An interesting question in this connection is the choice of the biodosimetric assay which yields the best results when carried out by inexperienced scorers. The aim of the present study was to compare the precision of setting up CA and MN dose response relationships by a group of such inexperienced scorers. To the best of our knowledge, the attempt to verify scoring quality by scorers 
who have not been thoroughly trained has not been investigated previously.

All data evaluated by non-experienced students displayed a clear dose-response dependence when analyzing $\mathrm{CA}$. When comparing each dose to $0 \mathrm{~Gy}$, they were all significantly discriminated, and also for some doses next to each other (1 vs 2 Gy and 3 vs 4 Gy). This strongly suggests that at least a broader difference in higher or lower dose received by an exposed person could be correctly distinguished by a nonexperienced scorer.

Overall, results of the students fitted well with those of the expert except for the two highest doses ( 3 and 4 Gy). The experienced scorer estimated significantly higher levels of aberrations at the end of the dose-response line, which may be explained by problems of proper identification of numerous aberrations by the students. Indeed, the largest discrepancy between the expert and the students was observed for breaks and gaps which suggests that students did not pay sufficient attention to counting chromosomal fragments.

The MN scores obtained by the experienced observer were consistent with student observations for the whole range of doses and the individual variation of the dose-response slope was smaller than that for CA. It can thus be concluded that the MN assay yields better results than the CA assay when carried out by inexperienced scorers.

Cytogenetic biological dosimetry is carried out in human peripheral blood lymphocytes (IAEA 2011). The present study was carried out with CHO-K1 cells and the question arises how far the reported results are relevant for biological dosimetry. The reason for choosing the $\mathrm{CHO}-\mathrm{K} 1$ cells was that the cells grow attached to a dish allowing shaking-off of mitotic cells. Consequently, following treatment with colcemid, the vast majority of cells dropped on microscopic slides are in mitosis and this facilitates scoring. Here it must be remembered that the scoring was carried out in the 
framework of the CELOD course where participants learn selected aspects of cellular radiation biology, with focus on cytogenetics. Given the limited course duration, it would take too long if the students had to score chromosomal aberrations in peripheral blood lymphocytes, where the mitotic index is generally low (IAEA 2011). With respect to the $\mathrm{MN}$ assay, $\mathrm{CHO}-\mathrm{K} 1$ cells have the advantage that all cells are proliferating, so the binucleation index is generally higher than in human peripheral blood lymphocytes. This also facilitates scoring. CHO-K1 cells have 21 long chromosomes and it can be expected that chromosomal aberrations are more easily recognized and quantified than in human peripheral blood lymphocytes. On the other hand, the low number of chromosomes (as compared to human cells) results in a relatively high number of complex aberrations per unit dose, making the identification of individual aberration types difficult. This may be the reason why the dose response curve for dicentric chromosomes (Figure 3c) was moderate. The quality of slides for scoring $\mathrm{MN}$ is not expected to differ between the cell types. Hence, it can be expected that the superiority of the MN assay over the CA assay reported here would be even more pronounced if the exercise had been carried out using human peripheral blood lymphocytes.

There are a few possibilities to improve the quality of the data being analyzed, if this experiment was to be carried out again. Firstly, to use a consistent sampling scale to reduce redundant data and give more informative results over a wider range (for example dose sampling with 1 Gy interval). Secondly, each observer performing at least 5 replicates (from the same experiment) for their allocated dose so the normality tests have some significance when trying to isolate observer bias, or using a smaller number of observers with higher replications for the same effect.

In conclusion, chromosomal aberration and micronuclei tests give the possibility to assess the dose-response even by non-experienced scorers. In the case of a 
radiological accident with a huge amount of data to be analyzed the support from people quickly trained is inestimable. Using basic statistical tools, it was shown that micronuclei are easier to be learned and scored giving a reasonable estimate of the cellular response after irradiation.

\section{Acknowledgements}

The work described in this paper has been partly supported by the European Commission, within the CONCERT project. This project has received funding from the Euratom research and training programme 2014-2018 under grant agreement No 662287. MG, AT, JŻ and BBW were supported by the Polish Founds of Science.

\section{Disclosure statement}

The authors report no conflict of interest. The authors alone are response for the content and writing of the paper.

References: see the journal's instructions for authors for details on style

Ainsbury EA, Bakhanova E, Barquinero JF, Brai M, Chumak V, Correcher V, Darroudi F, Fattibene P, Gruel G, Guclu I, Horn S, Jaworska A, Kulka U, Lindholm C, Lloyd D, Longo A, Marrale M, Monteiro GO, Oestreicher U, Pajic J, Rakic B, Romm H, Trompier F, Veronese I, Voisin P, Vral A, Whitehouse CA, Wieser A, Woda C, Wojcik A, Rothkamm K. 2011. Review of retrospective dosimetry techniques for external ionising radiation exposures. Radiat Prot Dosimetry 147: 573-592.

Ariyoshi K, Miura T, Kasai K, Nakata A, Fujishima Y, Shinagawa M, Kadono K, Nishimura M, Kakinuma S, Yoshida MA.: Age dependence of radiation-induced genomic instability in mouse hematopoietic stem cells. Radiation Research. 2018;190(6):623-633. doi: 10.1667/RR15113.1 
Ariyoshi K, Miura T, Kasai K, Nakata A, Fujishima Y, Yoshida MA.: Radiationinduced bystander effect in large Japanese field mouse (Apodemus speciosus) embryonic cells. Radiation and Environmental Biophysics. 2018;57(3):223-231. doi: 10.1007/s00411-018-0743-8

Ariyoshi K, Miura T, Kasai K, Fujishima Y, Oshimura M, Yoshida MA.: Induction of Genomic Instability and Activation of Autophagy in Artificial Human Aneuploid Cells. Mutation Research - Fundamental and Molecular Mechanisms of Mutagenesis. 2016;790:19-30. doi: 10.1016/j.mrfmmm.2016.06.001

Bender MA, Gooch PC. 1962. Persistent chromosome aberrations in irradiated human subjects. Radiation Research 16: 44-53.

Bickel PJ, Freedman DA. 1981. Some asymptotic theory for the Bootstrap, The Annals of Statistics 6: 1196-1217

Blakely WF, Carr Z, Chu MC, yal-Drager R, Fujimoto K, Hopmeir M, Kulka U, LillisHearne P, Livingston GK, Lloyd DC, Maznyk N, Perez MR, Romm H, Takashima Y, Voisin P, Wilkins RC, Yoshida MA. 2009. WHO 1st consultation on the development of a global biodosimetry laboratories network for radiation emergencies (BioDoseNet). Radiation Research 171: 127-139.

Cheng L, Lisowska H, Sollazzo A, Wegierek-Ciuk A, Stepien K, Kuszewski T, Lankoff A, Haghdoost S, Wojcik A. 2015. Modulation of radiation-induced cytogenetic damage in human peripheral blood lymphocytes by hypothermia. Mutat Res Genet Toxicol Environ Mutagen 793: 96-100.

Countryman PI, Heddle JA. 1976. The production of micronuclei from chromosome aberrations in irradiated cultures of human lymphocytes. Mutation Research 41: 321-332.

Fenech M. 2000. The in vitro micronucleus technique. Mutation Research 455: 81-95.

Fenech M. 2007. Cytokinesis-block micronucleus cytome assay. Nat Protoc 2: 10841104.

Fenech M, Morley AA. 1986. Solutions to the kinetic problem in the micronucleus assay. Cytobios 43: 233-246.

Fujishima Y, Kanahama S, Hagino S, Natsubori S, Saito H, Azumaya A, Ariyoshi K, Nakata A, Kasai K, Yamada K, Mariya Y, Yoshida MA, Miura T.: Influence of anticoagulants and storage temperatures on blood counts and mitotic index of blood samples collected for cytogenetic biodosimetry. International Journal of Radiation Biology. In press. doi: 10.1080/09553002.2019.1539882 
Greenland S, Senn SJ, Rothman KJ, Carlin JB, Poole C, Goodman SN, Altman DG. 2016. Statistical tests, P values, confidence intervals, and power: a guide to misinterpretations. Eur J Epidemiol 31: 337-350.

Holmquist GE. 1987. The magic of cytogenetic staining.

IAEA. 2011. Cytogenetic dosimetry: applications in preparedness for and response to radiation emergencies.

Johannes C, Obe G. 1991. Induction of chromosomal aberrations with the restriction endonuclease AluI in Chinese hamster ovary cells: comparison of different treatment methods. International Journal of Radiation Biology 59: 1379-1393.

Kulka U, Abend M, Ainsbury E, Badie C, Barquinero J, Barrios L, Beinke C, et al. 2017. RENEB - Running the European Network of biological dosimetry and physical retrospective dosimetry. International Journal of Radiation Biology 93: 2-14.

Lea DE, Catcheside DG. 1942. The mechanism of the induction by radiation of chromosome aberrations in Tradescantia. Journal of Genetics 44: 216-245.

Lloyd DC, Edwards AA, Moquet JE, Guerrero-Carbajal YC. 2000. The role of cytogenetics in early triage of radiation casualties. Applied Radiation and Isotopes 52: 1107-1112.

Müller WU, Streffer C. 1994. Micronucleus assays. Advances in Mutagenesis Research 5: $1-134$.

Romm H, Oestreicher U, Kulka U. 2009. Cytogenetic damage analysed by the dicentric assay. Ann Ist Super Sanita 45: 251-259.

Savage JRK. 1975. Classification and relationship of induced chromosomal structural changes. Journal of Medical Genetics 12: 103-122.

Takino S, Yamashiro H, Sugano Y, Fujishima Y, Nakata A, Kasai K, Urushihara Y, Kuwahara Y, Miura T, Fukumoto M.: Analysis of the effect of chronic and lowdose radiation exposure on spermatogenic cells of male large Japanese field mice (Apodemus speciosus) after the Fukushima Daiichi Nuclear Power Plant accident. Radiation Research. 2017;187(2):161-168. doi: 10.1667/RR14234.1

Tjio JH, Levan A. 1956. The chromosome number in man. Hereditas 42: 1-6.

Wojcik A, Lloyd D, Romm H, Roy L. 2010. Biological dosimetry for triage of casualties in a large-scale radiological emergency:capacity of the EU member states. Radiat Prot Dosimetry 138: 397-401. 
Wojcik A, Oestreicher U, Barrios L, Vral A, Terzoudi G, Ainsbury E, Rothkamm K, Trompier F, Kulka U. 2017. The RENEB operational basis: complement of established biodosimetric assays. International Journal of Radiation Biology 93: $15-19$. 
Appendix A

Table A1: Probability values for each dose data sets tested for Poisson (left column) and normal distribution (middle and right columns) calculated with Kolmogorow-Smirnow and Shapiro-Wilk tests.

\begin{tabular}{|c|c|c|c|}
\hline $\begin{array}{l}\text { Dose } \\
\text { (Gy) }\end{array}$ & $\begin{array}{l}\text { Probability of Poisson } \\
\text { distribution (Kolmogorow- } \\
\text { Smirnow) }\end{array}$ & $\begin{array}{l}\text { Probability of normality } \\
\text { (Kolmogorow-Smirnow) }\end{array}$ & $\begin{array}{l}\text { Probability of } \\
\text { normality (Shapiro- } \\
\text { Wilk) }\end{array}$ \\
\hline \multicolumn{4}{|c|}{$\mathbf{C A}$} \\
\hline $\mathbf{0}$ & 0.24 & 0.12 & 0.0011 \\
\hline 0.5 & 0.0049 & 0.28 & 0.021 \\
\hline 1 & 0.0044 & 0.086 & 0.00067 \\
\hline 2 & 0.0053 & 0.47 & 0.12 \\
\hline 3 & 0.00046 & 0.52 & 0.1 \\
\hline 4 & 0.0024 & 0.76 & 0.37 \\
\hline \multicolumn{4}{|c|}{ MN } \\
\hline $\mathbf{0}$ & 0.0020 & 0.0061 & 0 \\
\hline 0.5 & 0 & 0.048 & 0.00054 \\
\hline 1 & 0.00065 & 0.73 & 0.0064 \\
\hline 2 & 0.00057 & 0.66 & 0.046 \\
\hline 3 & 0.0038 & 0.17 & 0.00038 \\
\hline
\end{tabular}


Appendix B

Table B1. Fitted parameters ( $a$ and $b$ with its uncertainties $\Delta a$ and $\Delta b$ ) and goodness of the fit for dose-response curves based on chromosomal aberration scoring.

\begin{tabular}{|c|c|c|c|c|c|c|}
\hline Student ID & $a$ & $\Delta a$ & $b$ & $\Delta b$ & $\mathrm{Chi}^{2}$ & $\mathbf{R}^{2}$ \\
\hline An expert & 0.418 & 0.050 & -0.02 & 0.11 & 0.33 & 0.95 \\
\hline Mean & 0.186 & 0.081 & 0.118 & 0.126 & - & - \\
\hline 1 & 0.270 & 0.021 & 0.004 & 0.047 & 0.14 & 0.98 \\
\hline 2 & 0.123 & 0.013 & 0.091 & 0.030 & 0.26 & 0.96 \\
\hline 3 & 0.113 & 0.019 & 0.062 & 0.043 & 0.63 & 0.9 \\
\hline 4 & 0.102 & 0.054 & 0.26 & 0.12 & 3.17 & 0.47 \\
\hline 5 & 0.240 & 0.039 & -0.063 & 0.088 & 0.58 & 0.9 \\
\hline 6 & 0.177 & 0.018 & 0.100 & 0.040 & 0.24 & 0.96 \\
\hline 7 & 0.077 & 0.052 & 0.20 & 0.12 & 3.87 & 0.35 \\
\hline 8 & 0.092 & 0.021 & 0.062 & 0.046 & 1 & 0.83 \\
\hline 9 & 0.162 & 0.046 & 0.10 & 0.10 & 1.45 & 0.76 \\
\hline 10 & 0.204 & 0.046 & 0.32 & 0.10 & 1.02 & 0.83 \\
\hline 11 & 0.246 & 0.046 & 0.30 & 0.10 & 0.73 & 0.88 \\
\hline 12 & 0.033 & 0.011 & 0.182 & 0.024 & 1.82 & 0.7 \\
\hline 13 & 0.167 & 0.065 & 0.45 & 0.15 & 2.27 & 0.62 \\
\hline 14 & 0.242 & 0.049 & 0.34 & 0.11 & 0.85 & 0.86 \\
\hline 15 & 0.211 & 0.031 & 0.161 & 0.069 & 0.47 & 0.92 \\
\hline 16 & 0.215 & 0.034 & -0.009 & 0.077 & 0.56 & 0.91 \\
\hline
\end{tabular}




\begin{tabular}{|l|l|l|l|l|l|l|}
\hline $\mathbf{1 7}$ & 0.227 & 0.041 & 0.039 & 0.092 & 0.69 & 0.88 \\
\hline $\mathbf{1 8}$ & 0.1309 & 0.0091 & 0.027 & 0.020 & 0.11 & 0.98 \\
\hline $\mathbf{1 9}$ & 0.146 & 0.028 & 0.074 & 0.064 & 0.79 & 0.87 \\
\hline $\mathbf{2 0}$ & 0.122 & 0.011 & 0.042 & 0.025 & 0.2 & 0.97 \\
\hline $\mathbf{2 1}$ & 0.035 & 0.019 & 0.018 & 0.044 & 3.28 & 0.45 \\
\hline $\mathbf{2 2}$ & 0.264 & 0.025 & 0.172 & 0.056 & 0.2 & 0.97 \\
\hline $\mathbf{2 3}$ & 0.244 & 0.016 & 0.090 & 0.037 & 0.11 & 0.98 \\
\hline $\mathbf{2 4}$ & 0.197 & 0.070 & -0.02 & 0.16 & 2.02 & 0.66 \\
\hline $\mathbf{2 5}$ & 0.308 & 0.018 & -0.013 & 0.041 & 0.08 & 0.99 \\
\hline $\mathbf{2 6}$ & 0.124 & 0.049 & 0.14 & 0.11 & 2.33 & 0.61 \\
\hline $\mathbf{2 7}$ & 0.305 & 0.028 & 0.106 & 0.063 & 0.2 & 0.97 \\
\hline $\mathbf{2 8}$ & 0.315 & 0.050 & -0.04 & 0.11 & 0.56 & 0.91 \\
\hline $\mathbf{2 9}$ & 0.293 & 0.031 & 0.225 & 0.070 & 0.26 & 0.96 \\
\hline
\end{tabular}

Table B2. Fitted parameters ( $a$ and $b$ with its unceratinties $\Delta a$ and $\Delta b$ ) and goodness of the fit for dose-response curves based on scoring of micronuclei.

\begin{tabular}{|l|l|l|l|l|l|l|}
\hline Student ID & $\boldsymbol{a}$ & $\boldsymbol{\Delta} \boldsymbol{a}$ & $\boldsymbol{b}$ & $\boldsymbol{\Delta} \boldsymbol{b}$ & $\mathbf{C h i}^{\mathbf{2}}$ & $\mathbf{R}^{\mathbf{2}}$ \\
\hline An expert & $\mathbf{2 2 3}$ & $\mathbf{1 4}$ & $\mathbf{7}$ & $\mathbf{2 4}$ & $\mathbf{0 . 0 6}$ & $\mathbf{0 . 9 9}$ \\
\hline Mean & 172 & 62 & 36 & 74 & - & - \\
\hline $\mathbf{1}$ & 81.9 & 7.9 & 10 & 13 & 0.14 & 0.97 \\
\hline $\mathbf{2}$ & 167 & 26 & 73 & 43 & 0.33 & 0.93 \\
\hline $\mathbf{3}$ & 216 & 26 & -8 & 44 & 0.21 & 0.96 \\
\hline
\end{tabular}




\begin{tabular}{|l|l|l|l|l|l|l|}
\hline $\mathbf{4}$ & 169 & 25 & 24 & 43 & 0.32 & 0.94 \\
\hline $\mathbf{5}$ & 133 & 16 & 52 & 27 & 0.21 & 0.96 \\
\hline $\mathbf{7}$ & 150 & 31 & 52 & 52 & 0.57 & 0.89 \\
\hline $\mathbf{8}$ & 162 & 14 & 43 & 24 & 0.11 & 0.98 \\
\hline $\mathbf{9}$ & 191 & 13 & 16 & 21 & 0.07 & 0.99 \\
\hline $\mathbf{1 0}$ & 116.0 & 6.4 & 53 & 11 & 0.05 & 0.99 \\
\hline $\mathbf{1 1}$ & 180 & 32 & -1 & 55 & 0.45 & 0.91 \\
\hline $\mathbf{1 2}$ & 167.0 & 8.4 & 39 & 14 & 0.04 & 0.99 \\
\hline $\mathbf{1 3}$ & 203 & 86 & 77 & 140 & 1.74 & 0.65 \\
\hline $\mathbf{1 4}$ & 228 & 21 & -6 & 35 & 0.12 & 0.98 \\
\hline $\mathbf{1 5}$ & 159 & 18 & -1 & 31 & 0.19 & 0.96 \\
\hline $\mathbf{1 6}$ & 182 & 13 & 64 & 22 & 0.07 & 0.99 \\
\hline $\mathbf{1 7}$ & 187 & 28 & 89 & 48 & 0.32 & 0.94 \\
\hline $\mathbf{1 8}$ & 109 & 14 & -23 & 24 & 0.25 & 0.95 \\
\hline $\mathbf{1 9}$ & 154 & 14 & 14 & 23 & 0.12 & 0.98 \\
\hline $\mathbf{2 0}$ & 197 & 13 & -15 & 23 & 0.07 & 0.99 \\
\hline $\mathbf{2 1}$ & 373 & 34 & -51 & 57 & 0.12 & 0.98 \\
\hline $\mathbf{2 2}$ & 199 & 12 & 47 & 21 & 0.06 & 0.99 \\
\hline $\mathbf{2 3}$ & 192 & 17 & 9 & 29 & 0.12 & 0.98 \\
\hline $\mathbf{2 4}$ & 189 & 12 & 28 & 21 & 0.06 & 0.99 \\
\hline
\end{tabular}




\begin{tabular}{|l|l|l|l|l|l|l|}
\hline 26 & 189 & 23 & -17 & 39 & 0.21 & 0.96 \\
\hline
\end{tabular}


Table 1. Coefficients of variation for each aberration type calculated from results of all scorers (symbols from the first row are described in Fig. 1).

\begin{tabular}{|l|l|l|l|l|l|l|l|l|l|l|}
\hline & B & b & dic & R & M & m & IE & SU & G/g & All \\
\hline CV & 122 & 91 & 122 & 120 & 131 & 120 & 114 & 157 & 161 & 58 \\
\hline
\end{tabular}

Table 2. Coefficients of variation (CV) calculated for the fitted slope parameters in the chromosomal aberration (CA) and micronucleus (MN) tests.

\begin{tabular}{|l|l|l|}
\hline & CA & MN \\
\hline $\mathrm{CV}$ & $44 \pm 19$ & $29.4 \pm 8.6$ \\
\hline
\end{tabular}

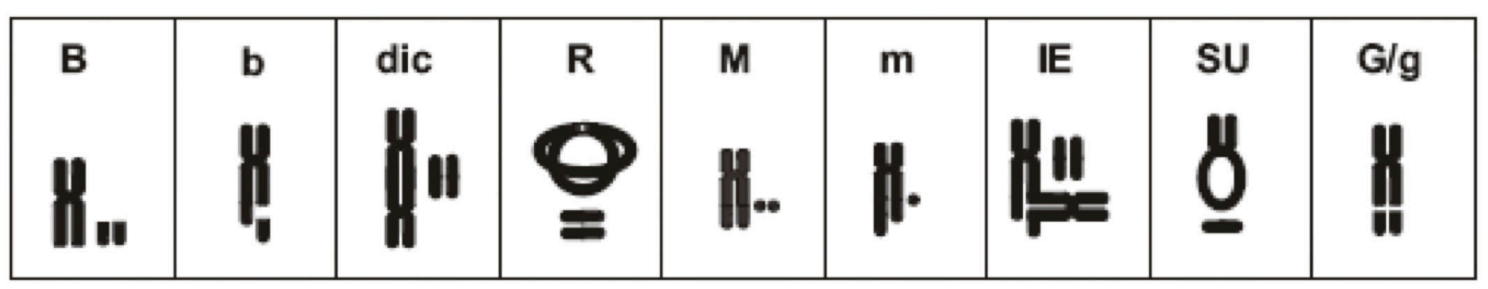

Figure 1. Types of analysed chromosomal aberrations (B - chromosome break, b chromatid break, dic - dicentric, $\mathrm{R}$ - ring, $\mathrm{M}$ - chromosome minute, $\mathrm{m}$ - chromatid minute, IE - interchromosomal exchange, SU - sister union, G/g - chromosome/chromatid gap). 


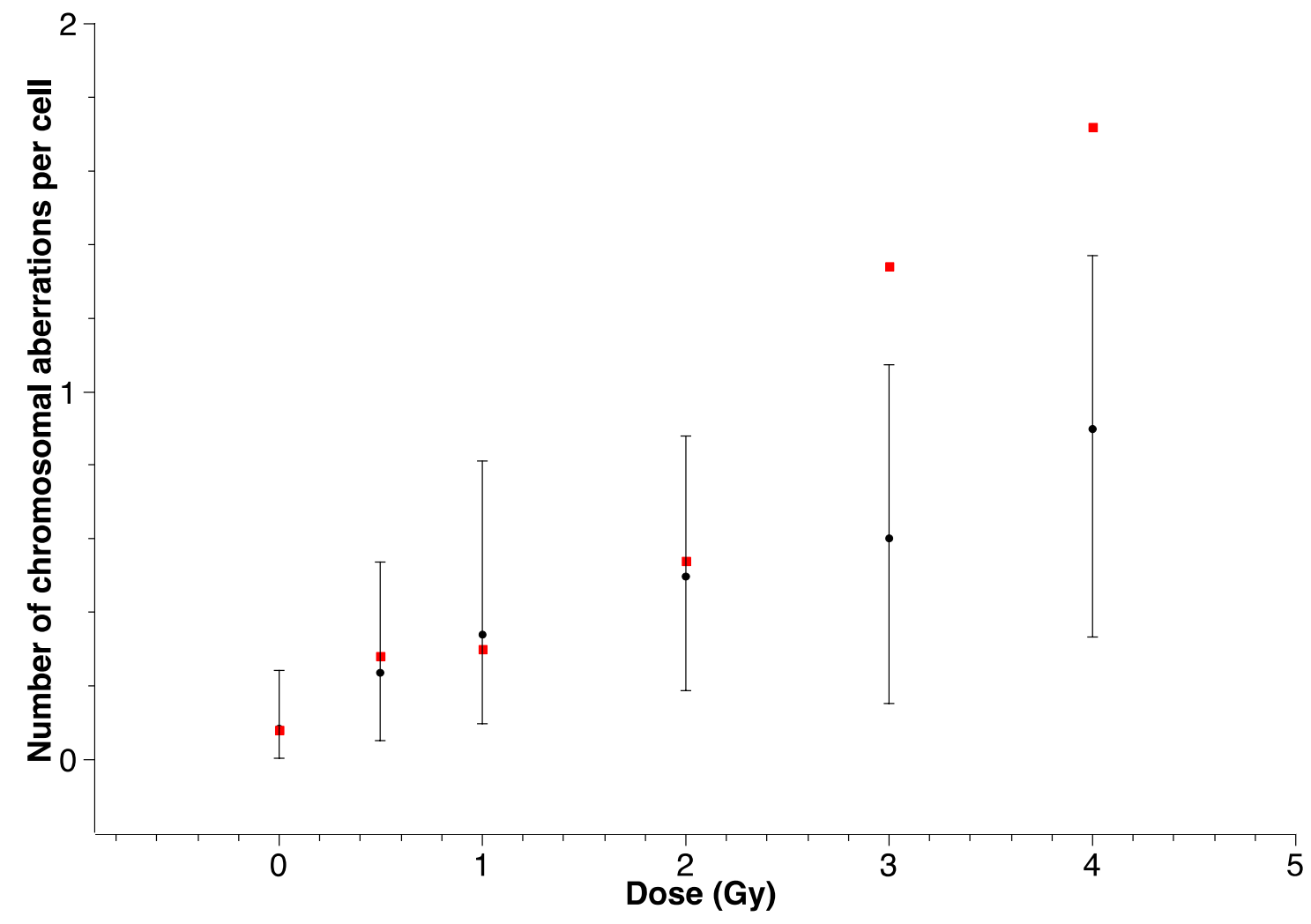

Figure 2. Comparison between the expert scores (red points) and observer scores of chromosomal aberrations with corresponding 95\% CI (black). 

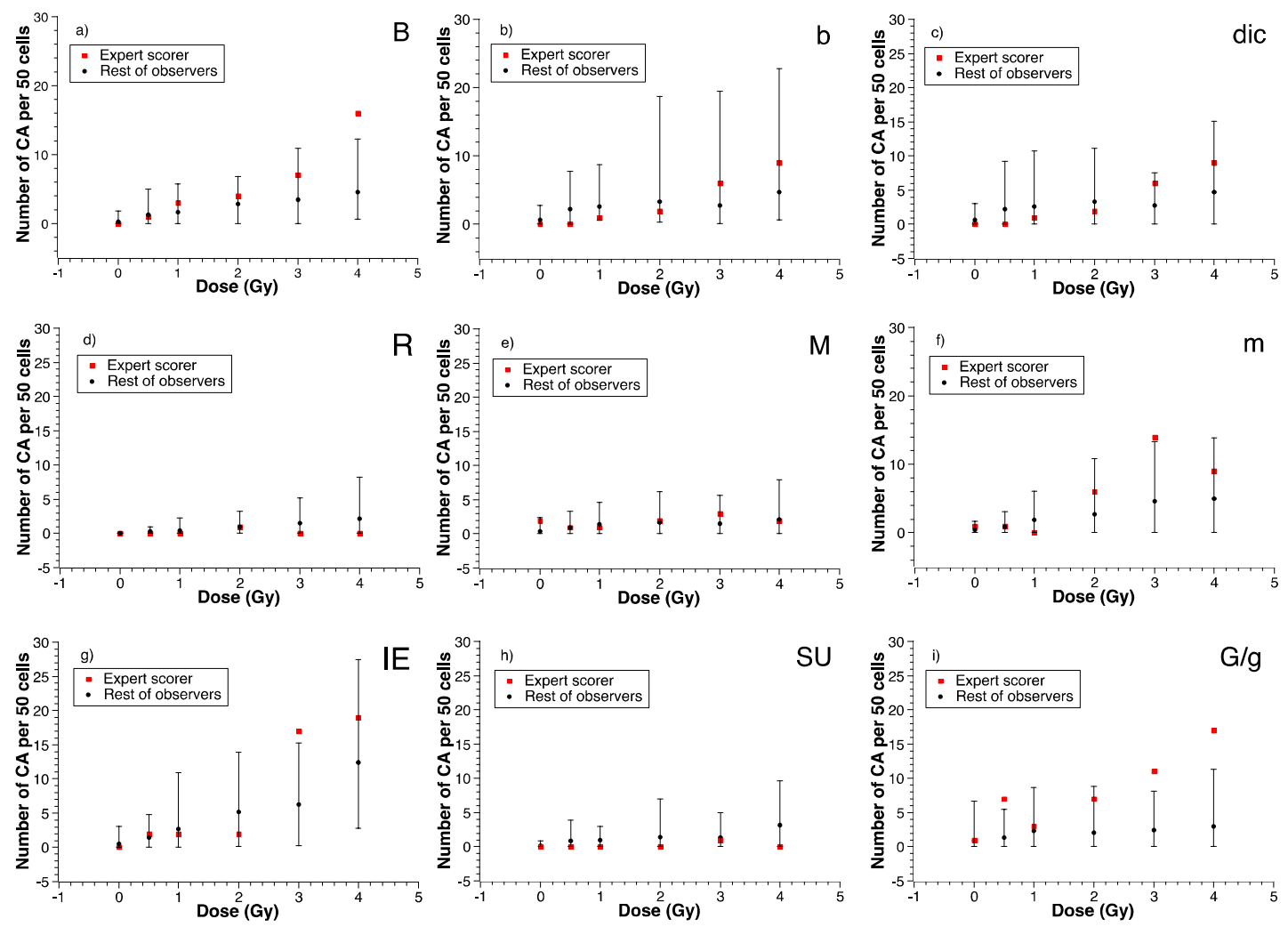

Figure 3. Comparison between the expert scores (red points) and observers (black) for each type of chromosomal aberration with corresponding $95 \%$ CI. 


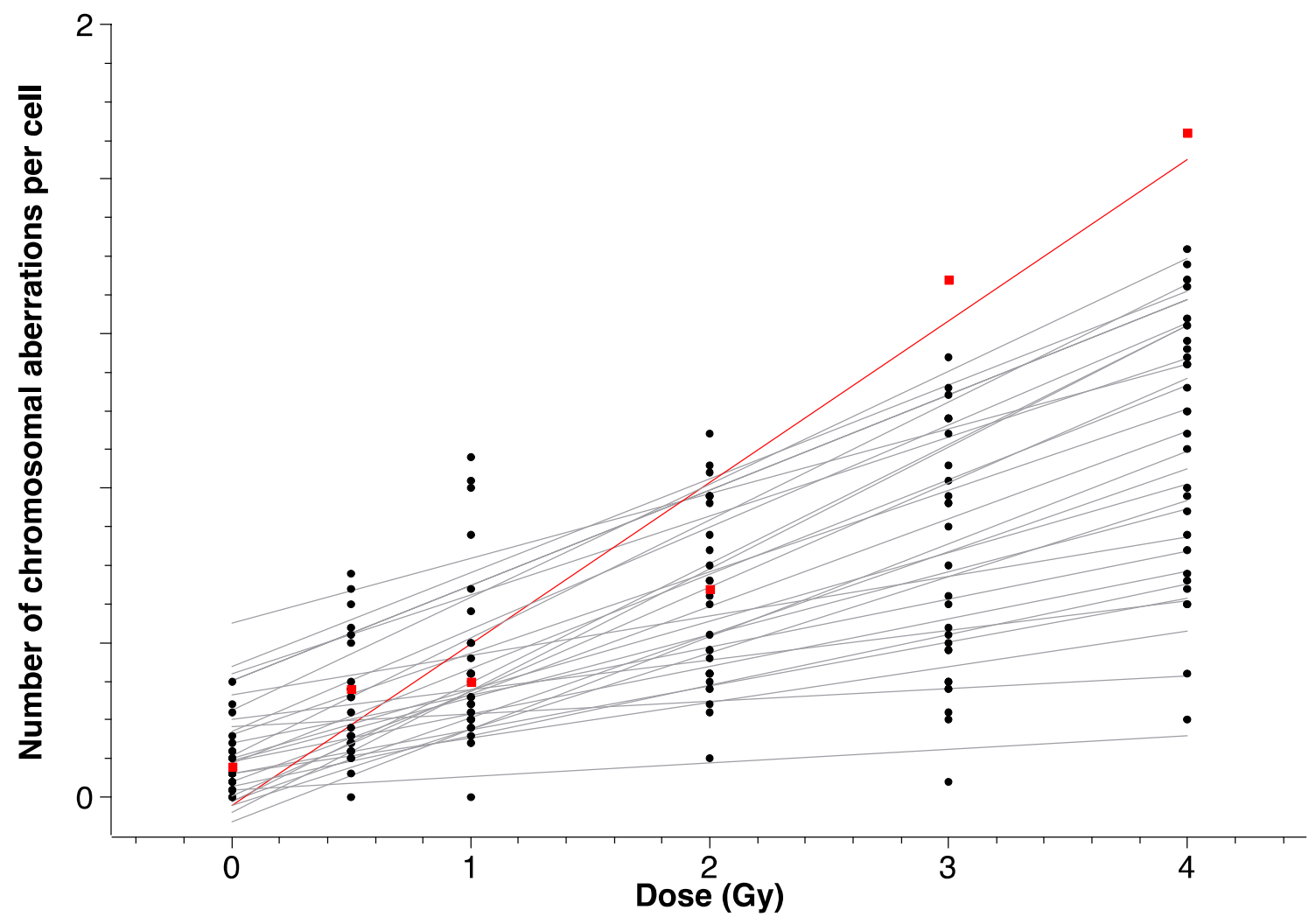

Figure 4. The dose-response regression lines (red - expert's, grey - students') superposed on the individual scores. 


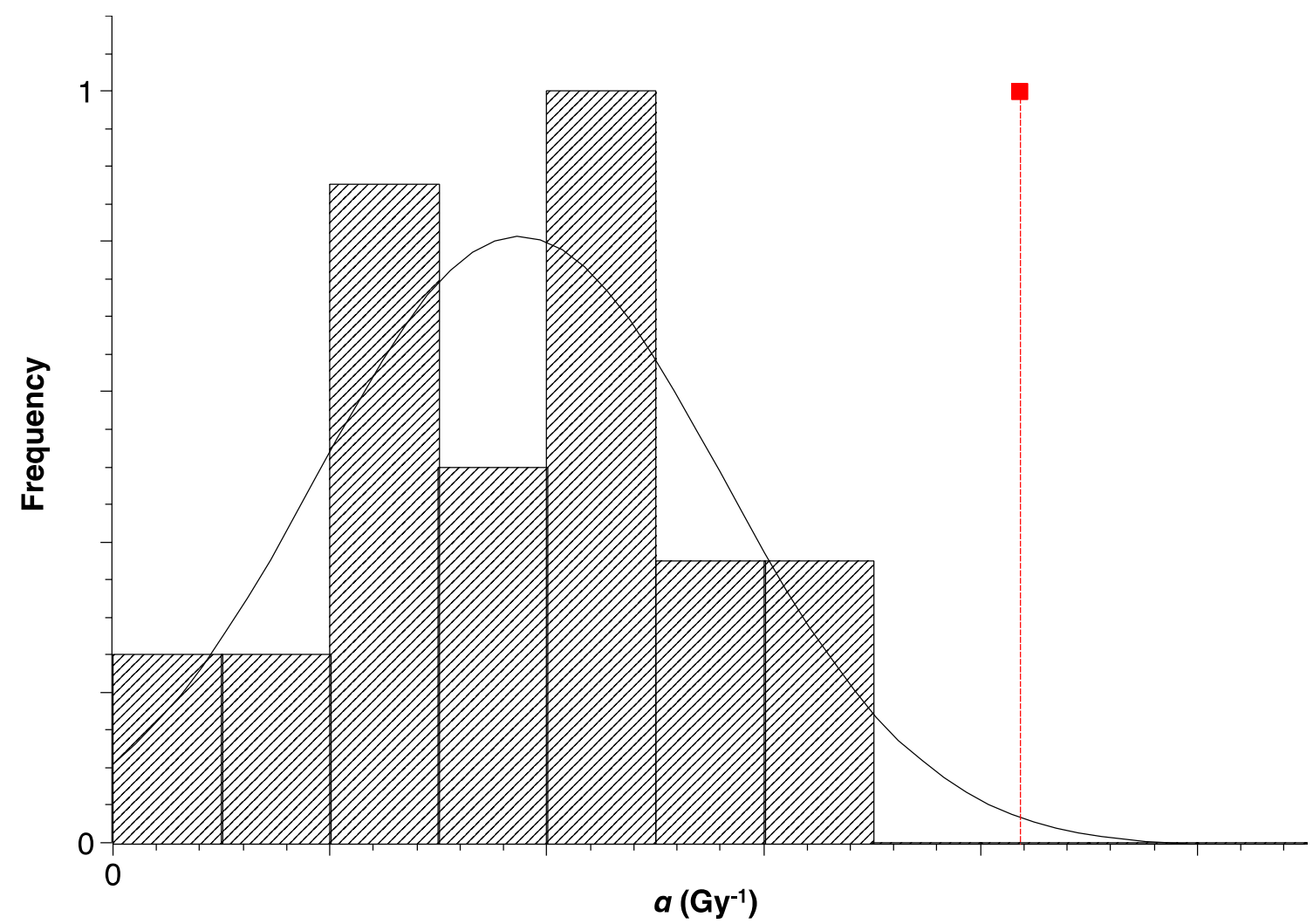

Figure 5. Distribution of slopes obtained for CA data set scored by each student with the expert's slope marked in red. 


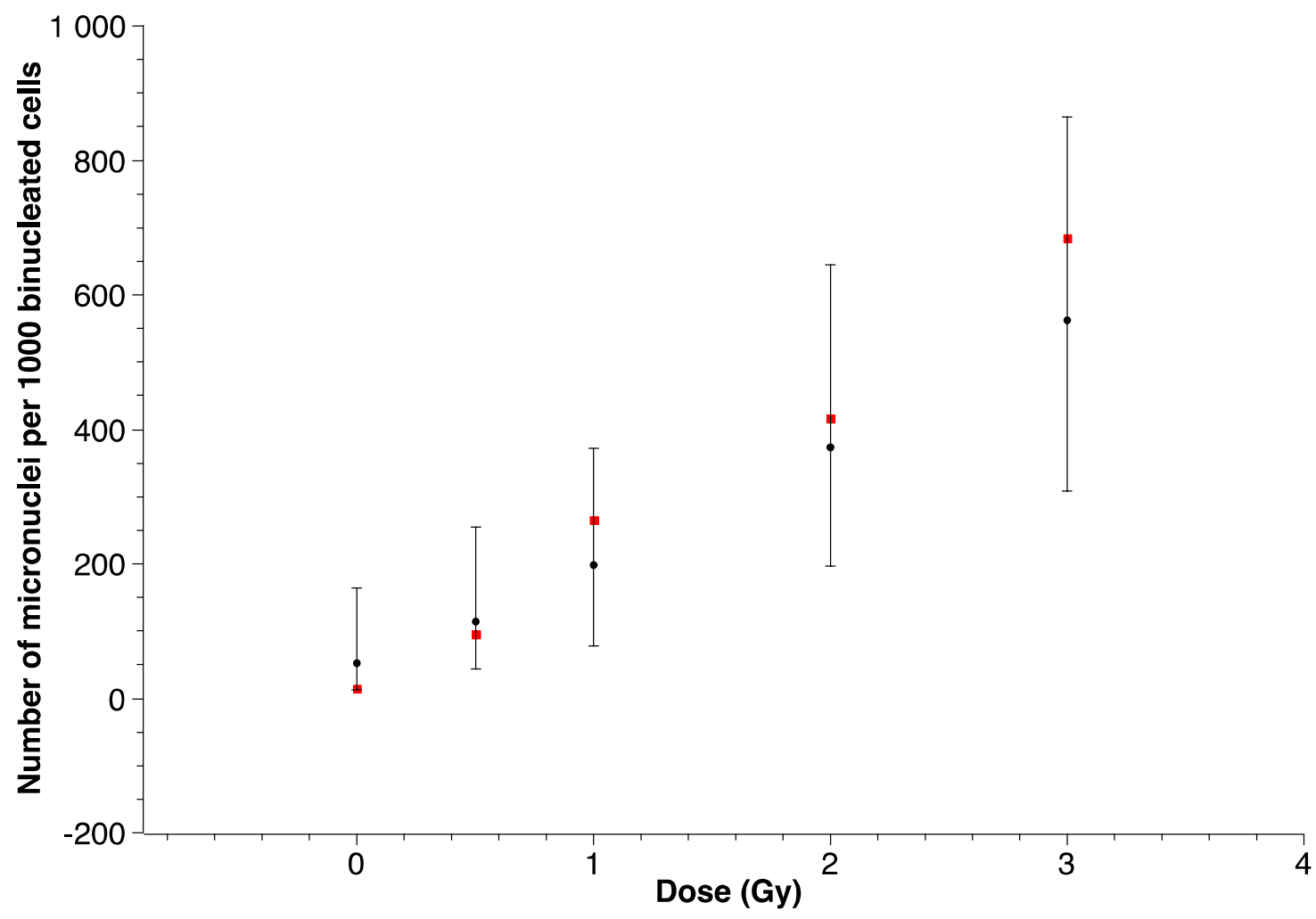

Figure 6. Comparison between observers (black points) and the expert scores (red points) of micronuclei. The $95 \%$ CI were calculated based on student data. 


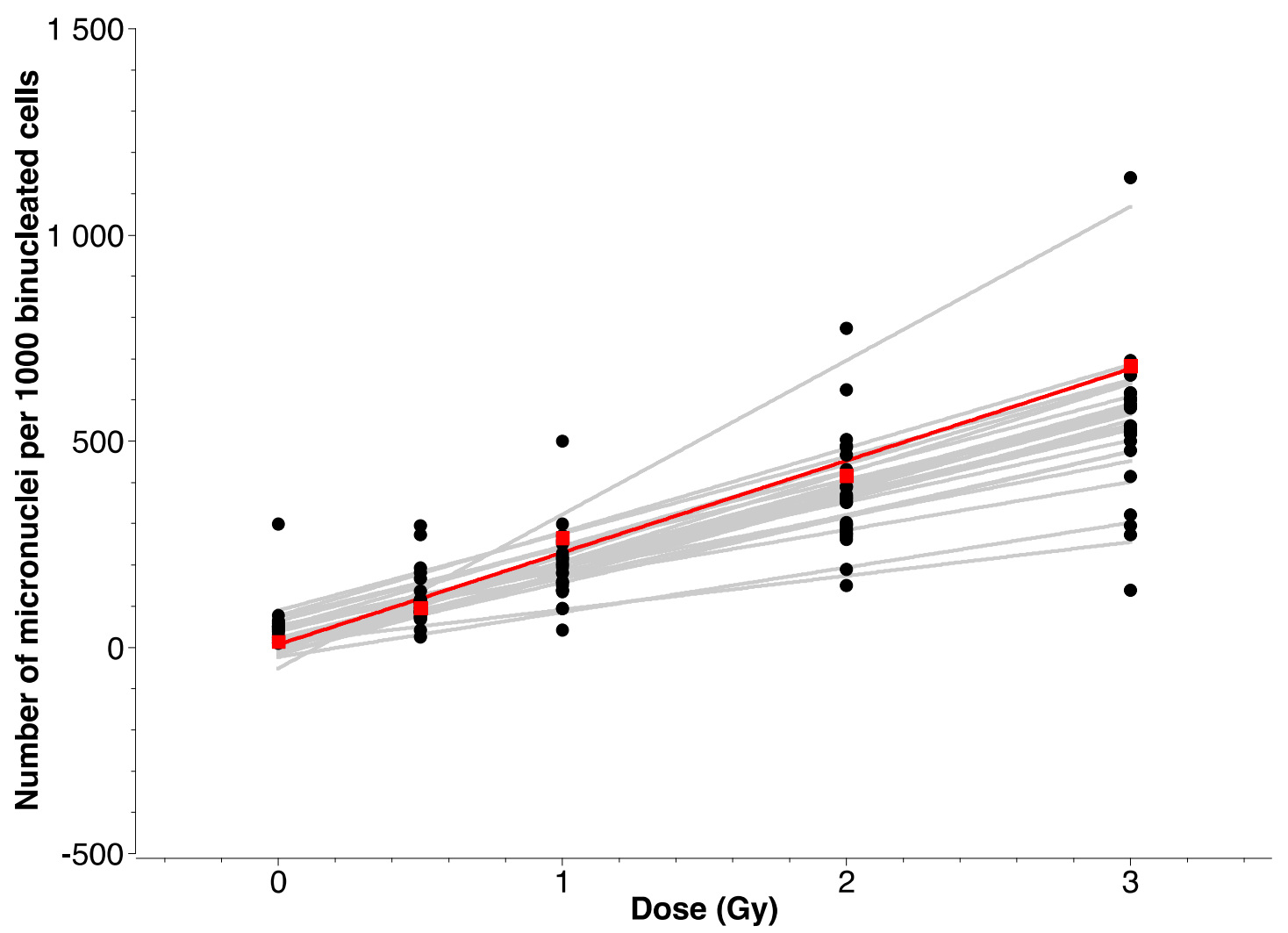

Figure 7. The dose-response curve obtained by an expert (red points) using MN assay. All student observations are shown in black with corresponding CI 95\% for each dose values. 


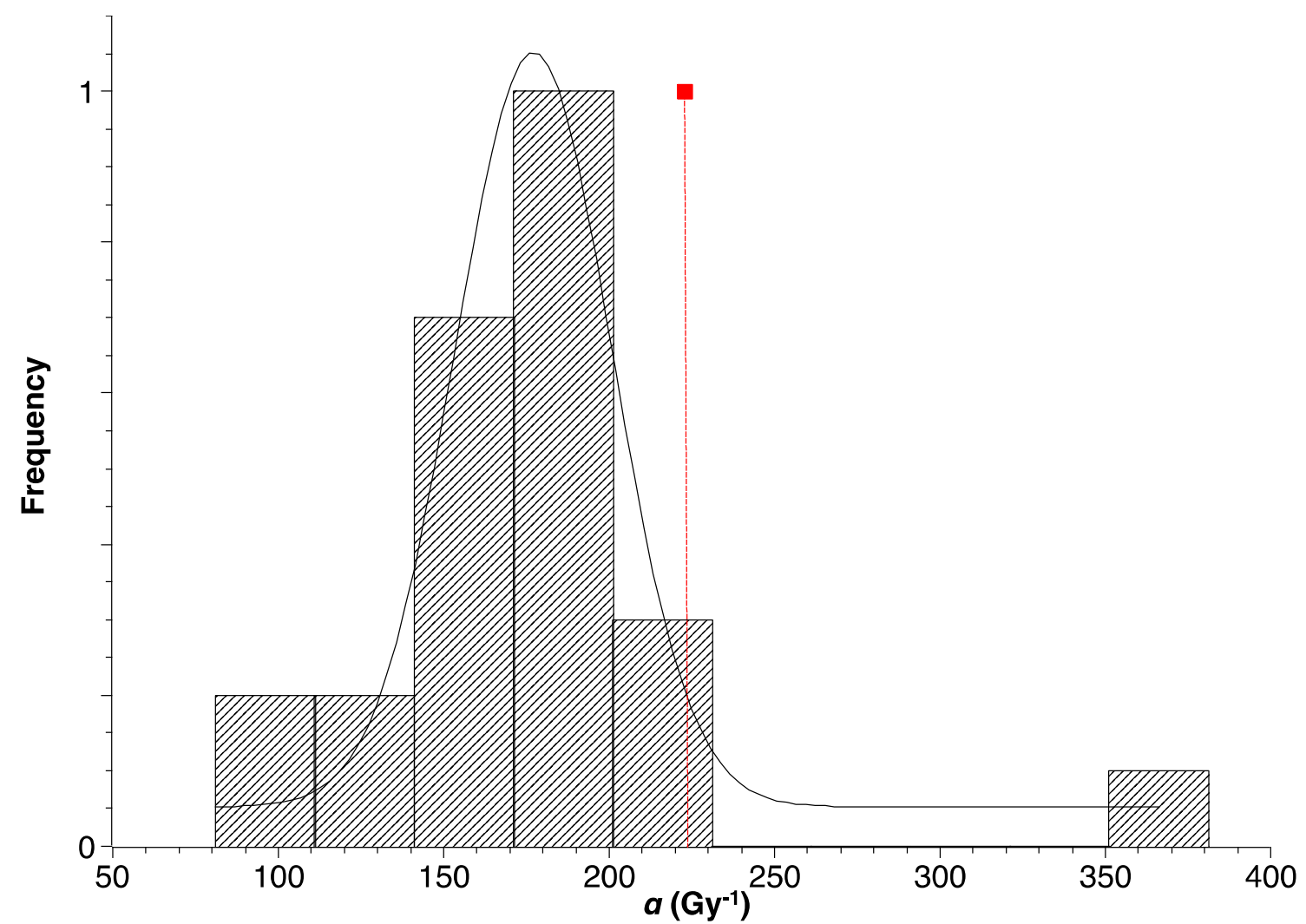

Figure 8. Histogram of dose-response slopes for MN data set scored by each student with the expert's slope marked in red. 